\title{
Molecular-weight dependence of interchain polaron delocalization and exciton bandwidth in high-mobility conjugated polymers
}

\author{
Jui-Fen Chang, ${ }^{1}$ Jenny Clark, ${ }^{1}$ Ni Zhao, ${ }^{1}$ Henning Sirringhaus, ${ }^{1, *}$ Dag W. Breiby, ${ }^{2}$ Jens W. Andreasen, ${ }^{2}$ Martin M. Nielsen, ${ }^{2}$ \\ Mark Giles, ${ }^{3}$ Martin Heeney, ${ }^{3}$ and Iain McCulloch ${ }^{3}$ \\ ${ }^{1}$ Cavendish Laboratory, University of Cambridge, Madingley Road, Cambridge CB3 OHE, United Kingdom \\ ${ }^{2}$ Danish Polymer Centre, Ris $\phi$ National Laboratory, 4000 Roskilde, Denmark \\ ${ }^{3}$ Merck Chemicals, University Parkway, Chilworth Science Park, Chilworth, Southampton SO16 7QD, United Kingdom
}

(Received 18 January 2006; revised manuscript received 15 June 2006; published 21 September 2006)

\begin{abstract}
Interchain interactions have a profound effect on the optical as well as charge transport properties of conjugated polymer thin films. In contrast to oligomeric model systems in solution-deposited polymer thin films the study of such effects is complicated by the complex microstructure. We present here a detailed study of interchain interaction effects on both charged polarons as well as neutral excitons in highly crystalline, high-mobility poly-3-hexylthiophene (P3HT) as a function of molecular weight. We find experimental evidence for reduced exciton bandwidth and increased polaron delocalization with increasing conjugation length and crystalline quality. From comparative studies of field-effect transistor characteristics, film morphology, and optical properties our study provides a microscopic understanding of the factors which limit the charge transport in P3HT to field-effect mobilities around $0.1 \mathrm{~cm}^{2} / \mathrm{V} \mathrm{s}$, and which will need to be addressed to improve mobility further.
\end{abstract}

DOI: 10.1103/PhysRevB.74.115318

PACS number(s): 73.40.Qv, 72.80.Le, 73.61.Ph

\section{INTRODUCTION}

Semiconducting conjugated polymers are complex physical systems due to the complex microstructure of solutionprocessed polymer films with an inevitable presence of disorder combined with strong electron-electron and electronphonon interactions causing neutral and charged excitations to be localized excitons and polarons, respectively. Their optical as well as charge transport properties are expected to depend critically on a variety of factors, such as the strength of interchain interaction effects, the presence of intrachain conjugation defects and/or the presence of other structural or chemical defects. A particularly interesting question is how interchain interaction effects depend on the molecular weight of the polymer. Many studies on model oligomeric systems have been devoted to studying the dependence of interchain interactions on the molecular, ${ }^{1}$ but in polymers such effects are much more challenging to investigate because they require a detailed understanding of the complex microstructure of the polymer. On the other hand a better physical understanding of interchain interaction effects in polymers is essential as these materials are becoming an attractive class of materials for realizing field-effect transistor (FET) circuits on low-cost flexible substrates by solution processing and direct-write printing techniques. ${ }^{2-4}$ Much progress has been made recently on their device performance, in particular with regard to increases in performance and operational lifetime, as well as scientific understanding of the device physics of these materials. ${ }^{5}$ Interchain interactions affect performance parameters, such as the field-effect mobility, critically, as they can lead, for example, to a limited interchain delocalization of the polaronic charge carriers. ${ }^{6}$ However, a much better understanding of how these effects depend on key polymer properties, such as molecular weight, is required in order to understand the factors that currently limit mobilities and to make further progress.
A wide range of solution-processed polymers has been investigated in an effort to optimize mobility through variation of chemical structure and modification of microstructure in solid films. One of the highest mobility and most widely studied materials is poly(3-hexylthiophene) ( $\mathrm{P} 3 \mathrm{HT})$, which is a microcrystalline, self-organizing polymer. On a microscopic scale P3HT adopts a highly anisotropic lamellar microstructure that can be aligned parallel to the substrate plane, in which strong interchain interactions lead to mobilities as high as $0.1 \mathrm{~cm}^{2} / \mathrm{V} \mathrm{s}{ }^{6,7}$ The field-effect mobility depends critically on the orientation of the conjugated lamellae and $\pi$ - $\pi$ stacking with respect to the substrate which can be controlled by the degree of regioregularity ${ }^{6}$ and dielectric modifications. ${ }^{8}$ On a mesoscopic scale P3HT can be made to adopt well defined nanoribbons using various processing techniques. ${ }^{9-11}$ The mobility depends critically on manifold other factors such as molecular weight, interaction between polymer and solvent, and deposition conditions. ${ }^{12}$ Recent research by McGehee et al. ${ }^{13,14}$ and Neher et al. ${ }^{15}$ focusing on the effects of molecular weight (MW), has shown that MW is another dominant factor to affect mobilities by several orders of magnitude in a regime with mobilities less than $10^{-2} \mathrm{~cm}^{2} / \mathrm{V} \mathrm{s}$. They found that the mobility increases with MW in spite of reduced crystallinity at higher MW, indicating that charge transport depends on factors other than the degree of crystallinity. Two possible explanations were proposed respectively. McGehee et al. attributed the higher mobility in high MW molecules to better interconnectivity of the polymer network, whereas low MW molecules form highly crystalline but more isolated microstructures. Neher $e t$ al. suggested that the mobility-MW relationship is mainly due to backbone conformation, and presented evidence that larger intrachain ring torsions tend to be present in low MW P3HT reducing the effective conjugation lengths for charge hopping. However, to date a microscopic understanding of the dependence of interchain interactions on molecular 
TABLE I. Physical parameters of P3HT samples including weight-average molecular weight $(\mathrm{Mw})$, polydispersity (PD), and degree of polymerization (DP) calculated from the number-average molecular weight.

\begin{tabular}{cccc}
\hline \hline Sample & Mw $(\mathrm{kD})$ & PD & DP \\
\hline 1 & 15.4 & 1.5 & 60 \\
2 & 20.0 & 1.8 & 66 \\
3 & 22.0 & 1.6 & 82 \\
4 & 29.0 & 1.5 & 114 \\
5 & 42.0 & 1.8 & 138 \\
6 & 52.0 & 2.3 & 138 \\
7 & 76.0 & 1.7 & 277 \\
8 & 270.0 & 2.3 & 723 \\
\hline \hline
\end{tabular}

weight, and of the factors that limit the field-effect mobility in $\mathrm{P} 3 \mathrm{HT}$ to values around $0.1 \mathrm{~cm}^{2} / \mathrm{V} \mathrm{s}$ is still lacking, as most previous studies were performed in a mobility range of $10^{-3}-10^{-2} \mathrm{~cm}^{2} / \mathrm{V} \mathrm{s} .^{13-15}$

In this paper we present a systematic study of the correlation of the electrical, structural, and spectroscopic properties of $\mathrm{P} 3 \mathrm{HT}$ as a function of $\mathrm{MW}$ in a high-mobility regime from $10^{-3}$ to $10^{-1} \mathrm{~cm}^{2} / \mathrm{V} \mathrm{s}$. A broad range of $\mathrm{MW}$ from $15 \mathrm{kD}$ to $270 \mathrm{kD}$ is investigated. In Sec. II we describe the experiments. In Sec. III we report on transistor characterization and field-effect mobility as a function of MW. In Sec. IV we discuss the film microstructure on the basis of atomic force microscopy, x-ray diffraction and electron microscopy. A model for chain folding as a function of MW is presented. In Sec. $\mathrm{V}$ we apply optical absorption and emission as well as charge modulation spectroscopy to study the spectroscopic properties of both neutral excitons and charged polarons as a function of MW. This allows us to extract information about the effects of molecular weight on both interchain polaron delocalization and exciton bandwidth. The paper concludes by discussing the implications of our findings on the factors which currently limit the field-effect mobility in P3HT FETs to values around $10^{-1} \mathrm{~cm}^{2} / \mathrm{V} \mathrm{s}$.

\section{EXPERIMENTAL}

Regioregular P3HT was synthesized by the transition metal mediated cross coupling of a 2,5-dibromo 3-hexylthiophene monomer under a range of catalytic and coreagent conditions ${ }^{16}$ to yield systematically varying molecular weight polymer with a consistently high regioregularity. Here we use weight-average molecular weight unless stated elsewhere. Care was taken to ensure that the regioregularity of the $\mathrm{P} 3 \mathrm{HT}$ was consistent and high for all molecular weight ranges. All polymers were purified by conventional methods, achieving consistently low level of ionic impurities. The molecular weights listed in Table I were determined by gel permeation chromatography (GPC) in chlorobenzene against polystyrene standards. The degree of polymerization (DP) calculated from the number average molecular weight is between 60 and 723. The P3HT solutions were prepared at a concentration of $10 \mathrm{mg} / \mathrm{ml}$ in chlo- roform or 1,2,4-trichlorobenzene (TCB), and heated at $70{ }^{\circ} \mathrm{C}$ for $30 \mathrm{~min}$ before being filtered through a $0.45 \mu \mathrm{m}$ pore size PTFE membrane syringe filter. The solubility of P3HT with MW from $15.4 \mathrm{kD}$ to $76 \mathrm{kD}$ in chloroform and TCB is reasonably good ( $>2$ wt. $\%$ ), while more time is required to dissolve $270 \mathrm{kD}$ due to the decreased solubility.

The transistors for this MW study were fabricated in a bottom gate, bottom-contact configuration. A heavily $n$-doped $\mathrm{Si}$ wafer acts as a common gate contact and 200 $\mathrm{nm}$ thermally grown $\mathrm{SiO}_{2}$ as gate dielectric. In order to reduce the effect of contact resistance for short channel devices $(<5 \mu \mathrm{m})$, we carefully patterned a $\mathrm{Au}$ layer of 15-20 nm without using an adhesion layer of $\mathrm{Cr}$ or $\mathrm{Ti}$ on the substrates as S/D electrodes by conventional photolithography. In P3HT TFTs with pure gold contacts fabricated in this way voltage drops across the source-drain contacts are negligible. ${ }^{17}$ The channel lengths of devices are varied from $20 \mu \mathrm{m}$ to $2 \mu \mathrm{m}$, and the channel widths are fixed at $1 \mathrm{~cm}$. The patterned substrates were treated with hexamethyldisilazane (HMDS) to produce a hydrophobic surface. P3HT solutions were drop cast or spin cast at $1500 \mathrm{rpm}$. The devices were completed by annealing at $100{ }^{\circ} \mathrm{C}$ for $10 \mathrm{~h}$ under vacuum to remove residual solvent and unintended doping. All the solution preparation, device making, and $\mathrm{I}-\mathrm{V}$ measurements were performed in a nitrogen atmosphere.

Grazing incidence x-ray diffraction measurements were performed using the $z$-axis diffractometer at the beamline BW2 in HASYLAB. The incoming monochromatic beam $(\lambda=1.2398 \AA)$ impinged onto the sample surface at a grazing incidence angle of $0.16^{\circ}$, whereby the (background) scattering from the substrate is suppressed. The sample cell, made of x-ray transparent Kapton, was flushed with He to reduce background and sample damage. The scattered intensity was collected using a Cyberstar point detector. In-plane scans, having the scattering vector $\mathbf{q}$ essentially parallel to the substrate, are presented in terms of $q \equiv|\mathbf{q}|=4 \pi \sin \theta / \lambda$, where $\theta$ is half the total scattering angle $2 \theta$. "Rocking scans" for constant $q=0.38 \AA^{-1}$, corresponding to the P3HT 100 reflection, are given in terms of $\chi$, which denotes the angle between $\mathbf{q}$ and the substrate. For a constant incidence angle, $\chi=90^{\circ}$ cannot be reached. Note that for a low $q$ of $0.38 \AA^{-1}$ there is considerable diffuse scattering, especially near the incidence plane (i.e., for high $\chi$ ). The crystallite correlation lengths $\xi$ can be estimated by the approximate Scherrer formula, ${ }^{18} \xi=K \times 2 \pi / \mathrm{W}$ where $K \approx 0.9$ is a numerical constant and $\mathrm{W}$ is the peak width (FWHM) in units of $\AA^{-1}$, found by fitting Gaussians to the peaks. The instrumental broadening is small, and within the considerable uncertainty associated with the Scherrer formula.

For indexing the diffraction data, we employ the simplest primitive unit cell consistent with the diffraction data. This cell contains two monomers, is orthorhombic, and has $a$ $=16.8 \AA, b=3.83 \AA$, and $c=7.8 \AA{ }^{19}$ The main polymer chains run parallel to the $c$ axis. The alkyl side chains are directed along the $a$ axis, which gives rise to the $h 00$ "lamellae" reflections. " $\pi$ stacking" between neighboring chains takes place along the $b$ axis, giving rise to the 010 reflection. However, in some of the data, a tendency of peaking is also observed at about $1.018 \AA^{-1}$. A possible indexing of this 
peak is 011 by the unit cell suggested by Prosa et al.,${ }^{20}$ which is doubled in both $a$ and $b$ directions with respect to the primitive cell. However, we prefer to use the defacto standard primitive cell for the present indexing. It is important to note that P3HT is semi-crystalline, implying that despite a few strong diffraction features, the amorphous regions of the films strongly influence their physical properties including the diffraction patterns.

Optical absorption and emission spectra were measured on P3HT films spin cast onto Spectrosil glass substrates. Absorption spectra were obtained using a Hewlett Packard 8453 uv-vis spectrometer. The emission spectra were measured using an Oriel Instaspec IV Spectrograph coupled to an optical fiber bundle. Excitation was provided by a multiline Ar-ion laser tuned to $488 \mathrm{~nm}$. The samples were measured in vacuum and cooled to $10 \mathrm{~K}$ using a continuous flow Oxford Instruments $\mathrm{He}$ cryostat. The mid-IR charge modulation spectra (CMS) were measured using a Fourier transform infrared (FTIR) spectrometer on bottom gate, top-contact FET devices for different MW films spin cast from TCB solution on low-doped silicon wafers with a $200 \mathrm{~nm}$ gate oxide. The devices were prepared under the same conditions and with similar mobilities as standard bottom gate, bottom-contact FETs. The CMS spectrum was extracted from the difference of infrared transmission spectra with devices being dc biased alternately in the accumulation $\left(V_{g}=-30 \mathrm{~V}\right)$ and depletion $\left(V_{g}=10 \mathrm{~V}\right)$ regimes, taking typically approximately 20000 averages.

\section{TRANSISTOR CHARACTERIZATION}

Figure 1(a) shows the field-effect mobility versus MW extracted for devices of $20 \mu \mathrm{m}$ channel length. Three kinds of deposition conditions are investigated to control the degree of crystallinity of the polymers, namely chloroform spin casting, chloroform drop casting, and TCB spin casting. The mobilities are extracted from the saturation regime at drain voltage $V_{\mathrm{ds}}=-50 \mathrm{~V}$ by fitting the slope of the square root of the drain current $I_{d}$ in the saturation regime versus gate voltage $V_{g}$, and each data point represents the average mobility from at least four transistors. The mobility depends critically on the combined effects of solvent/deposition conditions and MW. In TCB spin cast devices, the optimized mobility increases sharply with MW from $10^{-3}(15.4 \mathrm{kD}$ and $20 \mathrm{kD})$ up to $10^{-1} \mathrm{~cm}^{2} / \mathrm{V} \mathrm{s}(52 \mathrm{kD})$, and then starts to level off for even higher MW. The MW dependence of mobilities in chloroform drop cast devices behaves in a similar manner but saturates at a value slightly lower than $10^{-1} \mathrm{~cm}^{2} / \mathrm{V} \mathrm{s}$. In contrast, in films spin coated from chloroform the mobility varies only little between $3 \times 10^{-3}$ to $10^{-2} \mathrm{~cm}^{2} / \mathrm{V} \mathrm{s}$ in a MW range of $15 \mathrm{kD}$ to $270 \mathrm{kD}$, but no systematic dependence on MW is found. The latter result is consistent with the MW studies by McGehee et al. based on rapid thin film formation (spin casting from chloroform) which showed that mobility increases by three to four orders in magnitude with MW below $15 \mathrm{kD}$ but less than one order from $15 \mathrm{kD}$ onwards. ${ }^{13}$ This implies that the mechanism which leads to the improvement of mobility with increasing chain length that is observed in highly crystalline films under slow film formation conditions is not
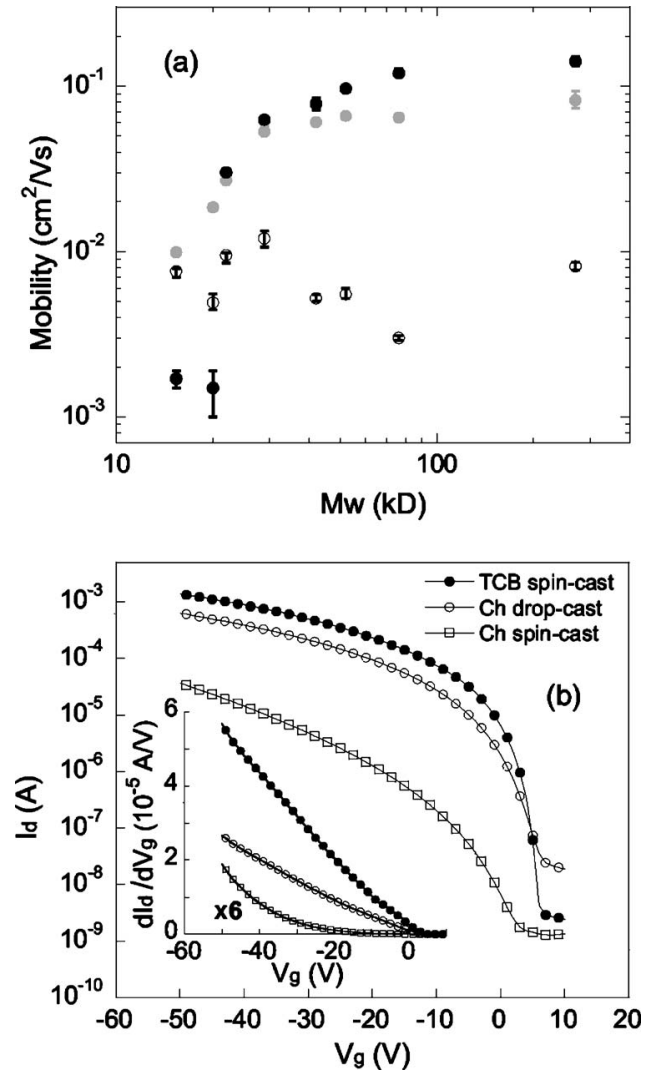

FIG. 1. (a) Mobility of $20 \mu \mathrm{m}$ long, $10 \mathrm{~mm}$-wide transistors in saturation regime $\left(V_{g}=-50 \mathrm{~V}, V_{\mathrm{ds}}=-50 \mathrm{~V}\right)$ as a function of weight average molecular weight for chloroform spin cast (open circle), chloroform drop cast (gray solid circle) and TCB spin cast (solid circle) depositions. (b) Transfer characteristics of the transistors with a molecular weight of $76 \mathrm{kD}$ at $V_{\mathrm{ds}}=-50 \mathrm{~V}$ for the three film depositions. Inset shows the transconductance of the devices.

active in more disordered microstructures of high molecular weight polymers $(>15 \mathrm{kD})$ produced by rapid film formation.

Figure 1(b) shows the transfer characteristics of transistors with MW of $76 \mathrm{kD}$ compared for the three film formation conditions. Under slow film formation conditions not only a higher mobility, but also a sharper turn-on behavior with a smaller subthreshold slope is observed. In addition, the transconductance $d I_{d} / d V_{g}$ exhibits a more linear dependence on $V_{g}$ above the threshold voltage $V_{t}$ which is indicative of a more gate voltage independent mobility, while for rapid film formation the mobility $\left(\propto d I_{d} / d V_{g}\right)$ is clearly gate voltage dependent. This suggests a better structural order in TCB spin cast and chloroform drop cast devices, and is corroborated by the higher degree of spectral broadening and blueshift of the absorption spectrum of films spin coated from chloroform (see Sec. V).

In contrast to the high MW regime where slowing down the film formation conditions improves the mobility, a different behavior is observed for MW less than typically 15-20 kD. Low MW samples show little improvement in mobility when slowing down the thin-film formation, probably indicative of polymer crystallites already being formed within a short time. The low mobilities in low MW films spin 


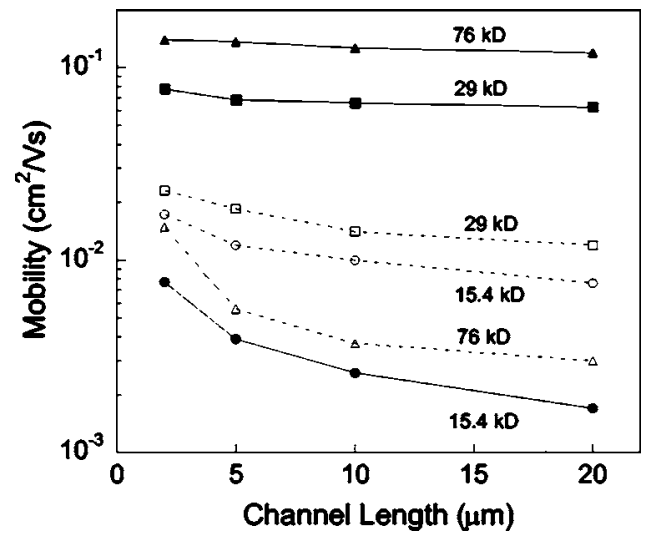

FIG. 2. Saturation mobility as a function of channel length. Open symbol represents for chloroform spin-cast devices and solid symbol for TCB spin-cast devices.

cast from TCB (MW $<15-20 \mathrm{kD})$ is peculiar. Their crystallinity is higher than those of chloroform cast films as suggested by the $\mathrm{x}$ ray and absorption spectra presented below. This result might be explained with the presence of more tie molecules linking crystallites in the less ordered films spin cast from chloroform, consistent with the model proposed in Ref. 13. However, for MW $>15-20 \mathrm{kD}$ there is clear evidence that slowing down the film formation, and in this way increasing the degree of crystallinity and structural anisotropy (as discussed below) leads to significant increase in mobility. The highest mobilities of $0.1 \mathrm{~cm}^{2} / \mathrm{V} \mathrm{s}$ and above can only be achieved in sufficiently high-MW P3HT with optimized crystallinity by spin casting from high-boiling solvents.

Figure 2 shows the channel length dependence of the saturation mobility for different MW polymers. Since P3HT transistors with pure $\mathrm{Au}$ electrodes exhibit negligible Schottky barrier for hole injection as evidenced by the scanning kelvin probe microscopy (SKPM), ${ }^{17,21,22}$ their contact resistance is assumed to be low enough that the channel length dependence of the mobility of P3HT can be extracted in this way. We observe that the saturation mobility of different MW generally increases as channel length decreases, which is opposite to the reduction of mobility caused by severe contact limited short-channel transistors with $\mathrm{Cr}-\mathrm{Au}$ or Ti-Au electrodes. ${ }^{23,24}$ The mobility is found to be more strongly channel-length dependent in low-mobility transistors, but little dependence is seen in highly crystalline, high-MW transistors. Figure 3 shows the output characteristics of $2 \mu \mathrm{m}$ - and $20 \mu \mathrm{m}$-channel TCB spin-cast transistors plotted on a current scale normalized by the channel length $L$. The $20 \mu \mathrm{m}$-channel transistors for both low (a) and high MW (b) polymers exhibit nearly ideal scaling characteristics. However, $2 \mu \mathrm{m}$-channel transistors exhibit not only a suppression of current by residual contact resistance in the linear regime but also a lack of saturation behavior due to shortchannel effects. ${ }^{25}$ These nonideal characteristics in short channels are more pronounced in low MW polymers, where the effective current exhibits a strongly superlinear behavior with source-drain voltage with a $5 \times$ higher apparent saturation mobility relative to the corresponding $20 \mu \mathrm{m}$-channel
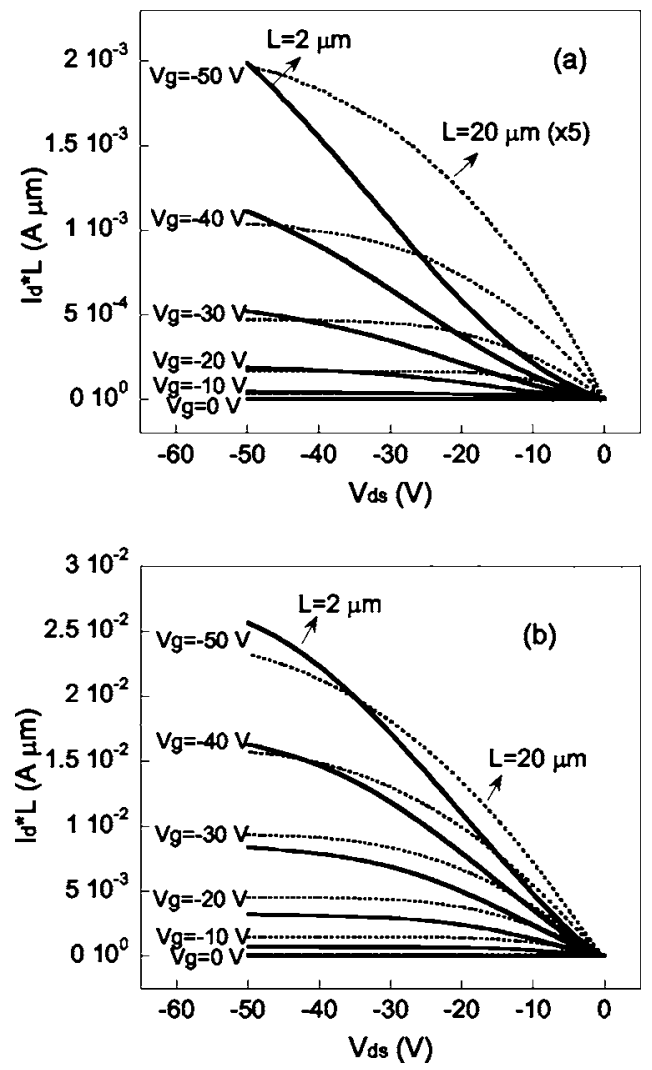

FIG. 3. Normalized output characteristics of $2 \mu \mathrm{m}$ and $20 \mu \mathrm{m}$ TCB spin-cast transistors for MW of (a) $15.4 \mathrm{kD}$ and (b) $76 \mathrm{kD}$.

device. The origin of the field-dependent mobility in many organic materials has been attributed to the electric field reducing the activation energy for charges hopping to neighboring sites in the field direction. ${ }^{26-28}$ Figures 2 and 3 therefore provide an indication that the low MW might be more disordered and have broader density of states leading to stronger field dependence of mobility for low MW polymers. ${ }^{29}$ In principle, one might expect an increase in mobility with reducing channel length as the channel length approaches the length scale of the polymer microcrystals (see below), i.e., charges need to cross fewer boundaries between microcrystals on their way from source to drain electrodes. That this is not observed in the high MW samples reported here suggests that the defects and disordered regions of the film which limit the mobility to values around $0.1 \mathrm{~cm}^{2} / \mathrm{V} \mathrm{s}$ must occur on a length scale significantly smaller than $2 \mu \mathrm{m}$.

\section{STRUCTURAL CHARACTERIZATION}

Diffraction data on the MW series show that for a fixed MW the field-effect mobility in a high mobility regime correlates closely with the degree of crystallinity and orientation of the microcrystalline lamellae with respect to the substrate plane. As shown in Fig. 4(a), all of the thin films prepared by the same processing method as used for the transistors exhibit a considerable degree of lamellar packing with the side chains [ $a$ axis, (100) diffraction] preferentially oriented perpendicular to the substrate, and the direction of $\pi$ - $\pi$ stacking 
(a)
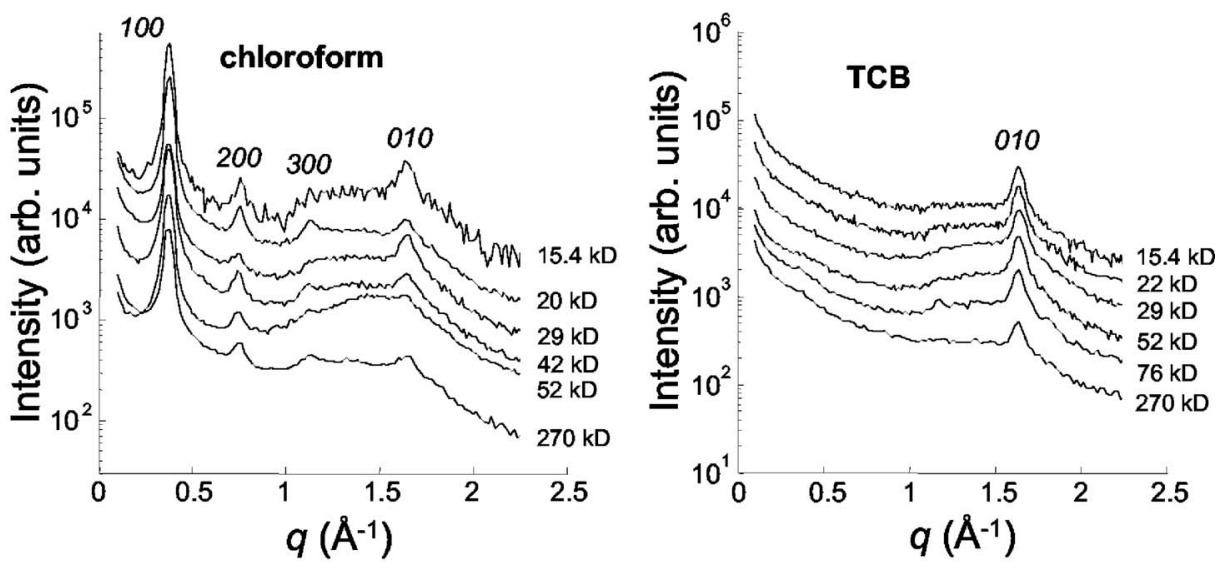

(b)
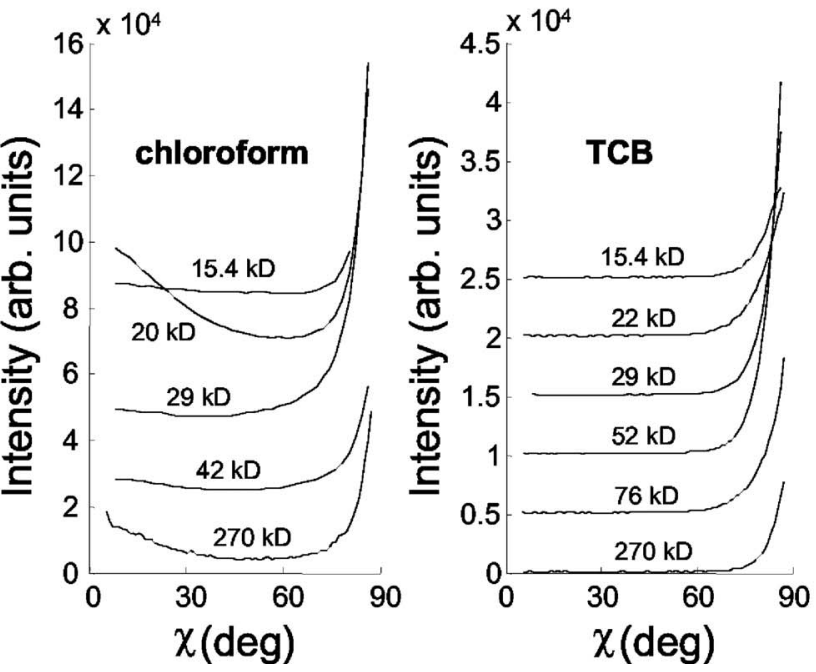

FIG. 4. (a) Grazing incidence x-ray diffraction measurements for P3HT thin films with different molecular weight spin cast from chloroform and TCB, with in-plane scattering geometry. The 100, 200, and 300 plane reflections are due to lamella layer structure and the 010 reflection is due to $\pi-\pi$ interchain stacking. The lowermost curve in each figure is the original data for $\mathrm{MW}=270 \mathrm{kD}$, and an offset of a factor 2 is applied to the other curves for reasons of clarity. The $\pi$-stack signal decreases with MW more apparently in chloroform samples while less in TCB samples. (b) Summary of the rocking scans for the 100 peak. The angle $\chi$ denotes the angle between $\mathbf{q}$ and the substrate (left diagram). An evident difference of anisotropy in crystalline textures can be seen between the chloroform and TCB samples.

[ $b$ axis, (010) diffraction] in the plane of the film. However, a significant difference in crystalline textures and structural anisotropy can be observed between the thin films spin-cast from TCB and chloroform over a wide range of MW. Similar effects have previously been reported for differences in stereoregularity, spin/drop casting, and choice of solvent. ${ }^{30-32}$ The polymers spin cast from chloroform tend to have a bimodal orientation distribution with a significant fraction of microcrystalline grains oriented with the $a$ axis in plane. ${ }^{31}$ This is also evidenced by rocking scans of the 100 reflection [Fig. 4(b)] which show that the crystallites exhibit a doublepeaked texture with side chains preferentially oriented either perpendicular or parallel to the substrate, but less preferrably at intermediate angles. In contrast, the TCB spin-cast films tend to form a single-peaked texture with only $\pi$ - $\pi$ stacking reflections observed in in-plane scans [Fig. 4(a)] and 100 reflection only present in a narrow orientation range around $\chi=90^{\circ}$. A high degree of preferred orientation for the 100 peak was measured in rocking scans with a FWHM of $\chi$ as low as $\sim 15^{\circ}$. As shown in our previous studies,${ }^{11}$ the preferential in-plane orientation of the microcrystalline lamellae in TCB spin-cast films supports efficient in-plane charge transport. In chloroform spin-cast films, the presence of microcrystalline grains with in-plane orientation of the side chains (and the thiophene rings), which are believed to constitute significant barriers for in-plane charge transport, provides an explanation for the significantly lower mobilities in such films compared to the more anisotropic films spin cast from TCB, since in microcrystalline lamellae oriented perpendicular to the substrate hopping across the alkyl side chains is likely to be required.

The intensity of the important " $\pi$-stacking" 010 reflection apparently decreases with MW for thin films spin cast from chloroform, which is in agreement with the observation by McGehee et al. and Neher et al. However, in the case of films spin cast from TCB, crystallinity as measured by the intensity of the 010 reflection varies only little with MW. Only the film with $270 \mathrm{kD}$ has a somewhat lower intensity of 
TABLE II. Table of in-plane peak widths. $W_{100}$ and $\xi_{100}$ in TCB samples are not measurable. The uncertainty in $W$ is less than $5 \%$. The relative uncertainty in $\xi$ (for comparisons between samples) is also less than $5 \%$, whereas the uncertainty on an absolute scale is large $(20-30 \%)$.

\begin{tabular}{ccccc}
\hline \hline Solvent & $W_{100}\left(\AA^{-1}\right)$ & $\xi_{100}(\AA)$ & $W_{010}\left(\AA^{-1}\right)$ & $\xi_{010}(\AA)$ \\
\hline chloroform & 0.05 & 113 & 0.10 & 57 \\
TCB & - & - & 0.07 & 81 \\
\hline \hline
\end{tabular}

the $\pi-\pi$ stacking. In terms of the orientation distribution we were unable to detect a systematic dependence on MW in either chloroform or TCB spun films (see rocking scan curves). For the chloroform case, the intermediate MW sample $(29 \mathrm{kD})$ appears to be an essentially single-peaked distribution, which likely accounts for its highest mobility observed among all the chloroform spun films. It is likely that the orientation distribution in chloroform spin-cast films is very sensitive to uncontrolled variations in film formation kinetics, and this might be why we have found it difficult to detect a systematic dependence with MW. As for TCB spun films, all exhibit a single-peaked orientational distribution and the x-ray peak widths and associated correlation lengths do not vary systematically with MW. Average values for each solvent are given in Table II. It is seen that for the chloroform sample, $\xi_{100}$ is about $113 \AA$, suggesting 5-8 repeat units along the $a$ axis. In the $\pi$ direction, the peaks are wider suggesting roughly 15 and 21 repeat units in the $b$ direction for chloroform and TCB, respectively. The TCB spin-cast films (except, to a degree, the $270 \mathrm{kD}$ sample) are all highly crystalline and anisotropic, and no clear correlation between microstructure and MW could be established that would help to explain the strong MW dependence of mobilities in TCB spin-cast films.

The previous studies based on rapid thin-film deposition claimed a highly ordered rodlike structure for relatively low MW films (3.2 kD) and an amorphous, nodulelike structure for high MW films. ${ }^{13}$ However, within our studied MW range very little difference can be resolved in the chloroform spin-cast films, all having an amorphous and granular surface topography in the AFM. In contrast, the TCB spin-cast films consist of macroscopic nanoribbon structures with length scale dependent on MW [Fig. 5(a)]. Other growth conditions for slow crystallization such as Langmuir-Blodgett method, ${ }^{33}$ precipitation in diluted poor solvents, ${ }^{34,35}$ post-annealing treatments, ${ }^{36}$ and solvent vapor-induced self-assembly ${ }^{10}$ also lead to such fibrillarlike microstructures. It is believed that the crystal growth of P3HT tends to favor one-dimensional crystals, ${ }^{37,38}$ with a length along the long axis extending from hundreds of nanometers to a few micrometers governed by the strong $\pi$-stacking interaction between chains. The length of the nanoribbons increases with MW significantly from $15 \mathrm{kD}$ to $29 \mathrm{kD}$, and soon reaches an apparent plateau in the intermediate MW range (between $29 \mathrm{kD}$ to $52 \mathrm{kD}$ ) followed by a relatively weak MW dependence. The nanoribbons of the highest MW polymer $(270 \mathrm{kD})$ are not obviously longer than those of $52 \mathrm{kD}$ polymer. Coincidently, the dependence of the length of nanoribbons on MW mirrors that of the (a)
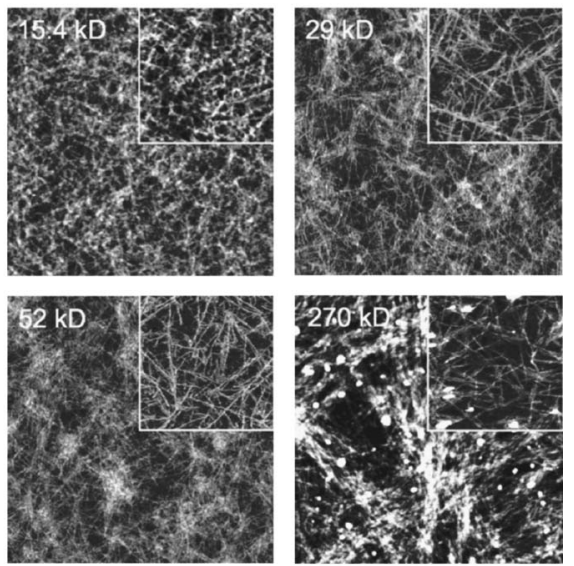

(b)

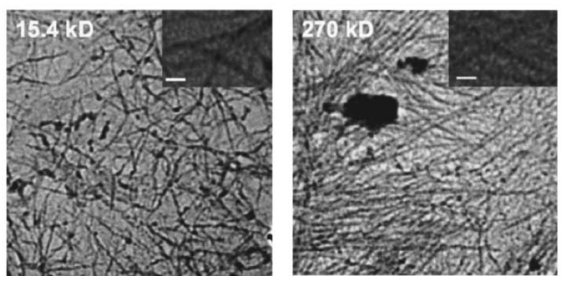

FIG. 5. (a) Tapping-mode atomic force microscopy images of TCB spin-cast transistors with different molecular weight. The image size is $5 \mu \mathrm{m} \times 5 \mu \mathrm{m}$. The formation of nanoribbons is observed, and the length of the nanoribbons increases rapidly between $15.4 \mathrm{kD}$ to $29 \mathrm{kD}$. To observe the nanoribbons more clearly we have also fabricated films on bare $\mathrm{SiO}_{2}$ (as opposed to HMDS treated $\left.\mathrm{SiO}_{2}\right)$ from more dilute solutions $(1 \mathrm{mg} / \mathrm{ml}$ as opposed to $10 \mathrm{mg} / \mathrm{ml}$ used for FET devices). These are shown in the insets. The isolated clusters in the $270 \mathrm{kD}$ sample are possibly related to the poorer solubility of this polymer. (b) TEM images of thin films drop cast from TCB solutions with concentration of $0.1 \mathrm{mg} / \mathrm{mL}$. The image size is $1.5 \mu \mathrm{m} \times 1.5 \mu \mathrm{m}$. The nanoribbons of $15.4 \mathrm{kD}$ and $270 \mathrm{kD}$ polymers appear with similar features as in TFT samples. The insets show nonuniformities along the axis of the nanoribbons. With the scale bar of $50 \mathrm{~nm}$, the estimated widths of nanoribbons are $15 \mathrm{~nm}$ for $15.4 \mathrm{kD}$ and $20 \mathrm{~nm}$ for $270 \mathrm{kD}$, respectively.

variation of mobilities with MW. We speculate that the longer nanoribbons provide elongated paths for charge transport along individual nanoribbons with fewer crossing points where charge need to hop from one nanoribbon to a neighboring one. Also longer nanoribbons appear to have a stronger tendency to orient parallel relative to each other, and this might facilitate the presence of tie molecules that can form a bridge between neighboring nanoribbons without significant chain twisting.

The physical dimension of nanoribbons resembles the whisker structures observed elsewhere, ${ }^{10,34}$ but surprisingly, we found that their height and the width are almost invariant within the MW range studied. The heights of nanoribbons measured by the AFM images for different MW exhibit statistics that reflect multiples of the $1.6 \mathrm{~nm}$ spacing between two conjugated lamellae. In average, a height of $3.2 \mathrm{~nm}$ and $4.8 \mathrm{~nm}$ appears most frequently, implying that stacking of two or three lamellae of conjugated backbones on top of 
each other is favored under the present crystal growth conditions. Moreover, the TEM images [Fig. 5(b)] reveal that the widths of nanoribbons for the lowest and highest MW films are almost identical, i.e., approximately $15 \mathrm{~nm}$ and $20 \mathrm{~nm}$, respectively. This finding is of particular interest since the two polymers have greatly different chain lengths $(282 \mathrm{~nm}$ for $270 \mathrm{kD}$ and $24 \mathrm{~nm}$ for $15.4 \mathrm{kD}$ ). Here the chain lengths are calculated from the number average molecular weight. Unlike the relatively low MW P3HT forming rigid nanorods composed of extended chain lamellae with a width approximate to single molecular length ${ }^{14}$ our result indicates that polymer chains above certain MW range tend to chain fold several times inside a nanoribbon. Some well-studied polymers such as polyethylene also show relatively little MW dependence of the crystal thickness under certain processing conditions. ${ }^{39-41}$

According to the molecular packing in $\mathrm{P} 3 \mathrm{HT}$ whiskers,${ }^{34}$ the polymer chains are oriented perpendicular to the nanoribbon axis. The contour length $L$ of a polymer chain which folds back and forth through a sequence of 2-3 conjugated lamellae on top of each other is approximately $30-60 \mathrm{~nm}$. This is assuming that chain folding occurs from one conjugated layer in the nanoribbon into the one on top or below it involving a sequence of thiophene rings in cis conformation, but not into another position in the same conjugated lamella. The contour length is comparable to the extended chain length of low MW polymers (e.g., $24 \mathrm{~nm}$ for $15.4 \mathrm{kD}$ and $32 \mathrm{~nm}$ for $22 \mathrm{kD}$ ) but greatly shorter than the extended chain length of high MW polymers (e.g., $108 \mathrm{~nm}$ for $76 \mathrm{kD}$ and $282 \mathrm{~nm}$ for $270 \mathrm{kD}$ ).

Based on the above observations we propose a simplified chain folding scheme as a function of MW. As illustrated in Fig. 6, the eight MW samples investigated here between $15.4 \mathrm{kD}$ and $270 \mathrm{kD}$ are classified into three groups depending on their chain lengths relative to the contour length $(L)$. For the low MW group (MW $<22 \mathrm{kD}$ ) with chain lengths shorter than $L$, there is a higher statistical probability for more than one molecule folded into a particular cross section perpendicular to the axis of the nanoribbon, leading to defective crystals with a disruption of the crystalline packing in the vicinity of chain ends and associated chemical defects and impurities [Fig. 6(a)]. We speculate that the presence of such defect sites contribute to the low mobility in low MW polymers. In the intermediate MW group ( $29 \mathrm{kD}-52 \mathrm{kD}$ ) the chain lengths are sufficiently long that polymer chains can fold into lamellae without chain ends being present inside the crystals [Fig. 6(b)]. In this regime the crystal quality of the nanoribbons is expected to be highest with the lowest number of defect sites, leading to the mobility increasing dramatically from low to intermediate MW regimes. Once the chain lengths significantly exceeds $L(76 \mathrm{kD}$ and $270 \mathrm{kD}$ ), the polymer chain needs to either reenter the same nanoribbon at some distance along the axis of the ribbon or fold into another nanoribbon [Fig. 6(c)]. In this way, the number of tie molecules linking different nanoribbons is enhanced, particularly, if the nanoribbons are preferentially aligned parallel to each other or at a small angle. On the other hand, the more pronounced polymer entanglement during growth, the possible presence of branches in the highest MW polymers, and the percentage of chain segments located (a)

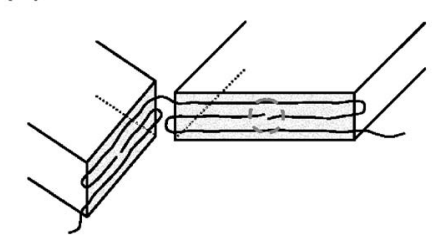

(b)
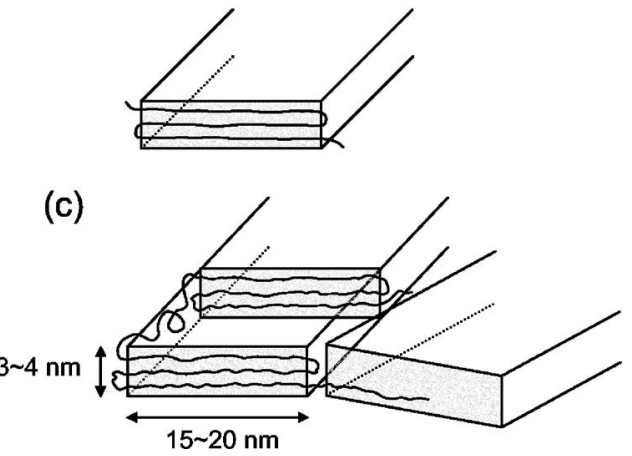

FIG. 6. Different chain folding schemes in nanoribbons depending on the chain lengths of polymers. Cross-sectional plane normal to the nanoribbon axis in low MW polymers $(<22 \mathrm{kD})$ (a) comprises more than one polymer leading to a higher population of chain ends inside the nanoribbons. Intermediate MW polymers (29 kD-52 kD) (b) have less chain end defects. High MW polymers $(76 \mathrm{kD}$ and $270 \mathrm{kD})$ (c) might fold into different sections of the same nanoribbon or extend to neighboring nanoribbons.

on the disordered surface of nanowires might lead to an overall higher degree of conformational disorder, producing chains containing kinks, bends, and twists arising from the folding kinetics. Consequently, we expect the highest MW nanoribbons to have a lower crystal quality than those in the intermediate MW group, which is evidenced by the decreased ordering of $\pi-\pi$ stacking in the $270 \mathrm{kD}$ sample, and is corroborated by the interpretation of the optical spectroscopy results reported below. Such kinetically induced reduction in crystal quality for the high MW polymers may account for the fact that the mobility does not further increase with MW but saturates in this regime.

\section{SPECTROSCOPIC CHARACTERIZATION}

To study the effect of MW on the spectroscopic properties of neutral excitons we have performed optical absorption (Fig. 7) and emission measurements (Fig. 8). For thin films spin cast from chloroform, we mainly observe a regular redistribution of the intensity of the absorption features as a function of MW rather than a significantly blueshifted absorption in low MW polymers due to the distortion of the backbones as reported previously. ${ }^{15}$ This clearly demonstrates that the polymers with MW higher than $15 \mathrm{kD}$ have similar backbone conformations in solid films. The peak positions are roughly the same for the various MW studied, but the $0-0$ transition $(\sim 2.05 \mathrm{eV})$ previously shown to be correlated with the degree of crystallinity, ${ }^{42}$ slightly reduces in intensity with increasing MW. Compared to the relatively 

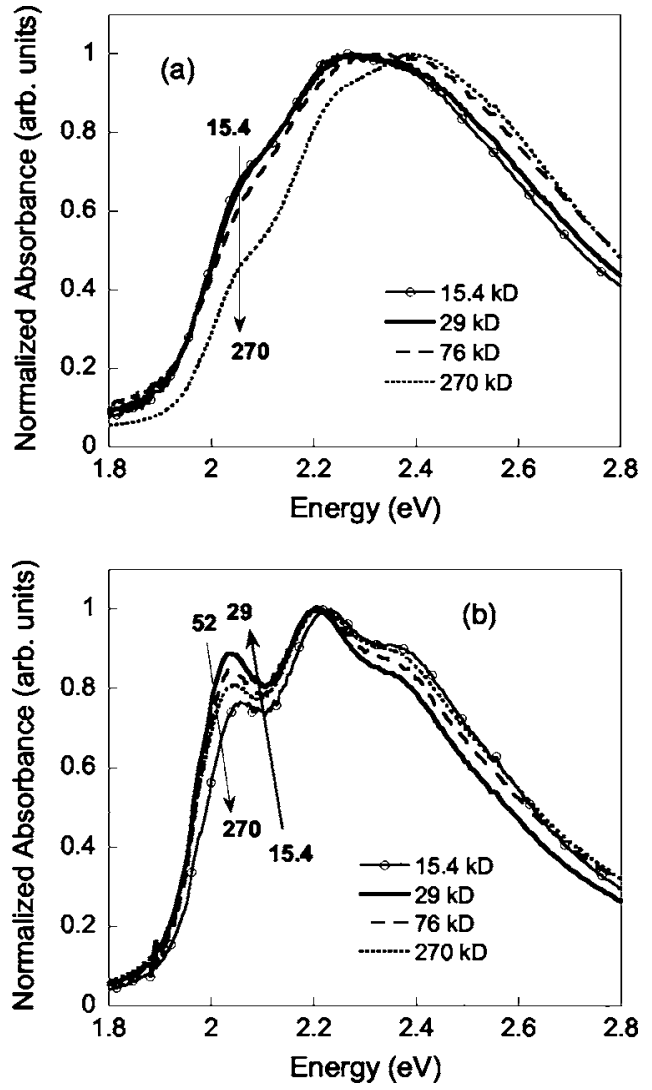

FIG. 7. Normalized absorption spectra of (a) chloroform spincast films and (b) TCB spin-cast films for different MW polymers. The arrows in (b) indicate the systematic variation in the intensity ratio between the 0-0 and 0-1 transition which increases with MW from low MW, reaches a maximum around $29-52 \mathrm{kD}$, and then decreases for $\mathrm{MW}>52 \mathrm{kD}$.

featureless spectra of chloroform spin-cast films, the more ordered TCB spin-cast films exhibit well structured $\pi$ - $\pi^{*}$ absorption with resolved transitions at $\sim 2.04, \sim 2.22$, and $\sim 2.36 \mathrm{eV}$ for the whole range of MW. We observe that the intensity ratio of the $0-0$ transition $(2.04 \mathrm{eV})$ to $0-1$ transition $(2.22 \mathrm{eV})$ is lowest in the lowest MW polymer, increases with MW up to the intermediate range $(29-52 \mathrm{kD})$, reaches a maximum around $29-52 \mathrm{kD}$ and then decreases again in the high MW range (76 kD and $270 \mathrm{kD}$ ) [Fig. 7(b)].

The optical properties of $\mathrm{P} 3 \mathrm{HT}$ are strongly influenced by the presence of interchain interactions. Recently, $\mathrm{Spano}^{43}$ has analyzed theoretically the first excited state in P3HT in terms of a weakly interacting $H$-aggregate type state. According to Kasha's rule, emission at low temperature comes from the lowest-energy excited state, which is symmetry forbidden with respect to the ground state for an $H$-aggregate arrangement, unless the presence of disorder relaxes the optical selection rules. Korovyanko et al. have also attributed the absorption and emission spectra of P3HT to an $\mathrm{H}$-aggregate-type state. ${ }^{44}$ They estimated an upper bound of the interchain coupling to be $\sim 250 \mathrm{meV}$, however, which would result in spectra showing little vibronic structure-not the case for P3HT.

Spano's model considers the weak exciton coupling regime for a lamellar aggregate in which interchain interac-

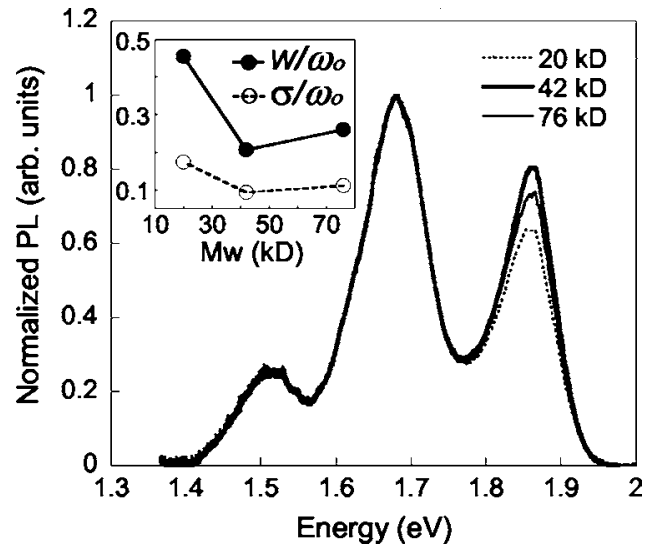

FIG. 8. Normalized PL spectra of TCB spin-cast films for different MW polymers at $10 \mathrm{~K}$. The inset shows the values of exciton bandwidth $W / \omega_{0}$ and disorder parameter $\sigma / \omega_{0}$ extracted from the $0-0$ to $0-1$ peak intensity ratio in the absorption and emission spectra.

tions give rise to an excitonic band with a bandwidth $W$ that is smaller than the energy $\hbar \omega_{0}$ of the vibrational mode coupled to the electronic transition. This is normally taken to be the $\mathrm{C}=\mathrm{C}$ stretch at around $1400 \mathrm{~cm}^{-1}$ for polythiophenes. ${ }^{42,45}$ The optical transition between the ground state and the lowest energy state in the exciton band is assumed to be symmetry forbidden, and the 0-0 transition in absorption is assumed to involve primarily a transition to the higher energy states in the exciton band. In emission the 0-0 transition can become weakly allowed as a result of disorder, which allows the forbidden transition from the state at the bottom of the exciton band to borrow oscillator strength from the transition at the top of the exciton band. Using Spano's model the degree of excitonic coupling can be quantitatively estimated, from the peak ratio of the $0-0$ transition and the $0-1$ transition $(\sim 2.25 \mathrm{eV})$ of the absorption spectrum. The exciton bandwidth $W$ (which is four times the interchain interaction energy for a one-dimensional linear aggregate $^{1,46}$ ) can be estimated from the following expression

$$
\frac{I_{a}^{0-0}}{I_{a}^{0-1}} \approx \frac{\left(1-0.24 W / \omega_{0}\right)^{2}}{\left(1+0.073 W / \omega_{0}\right)^{2}}
$$

Decreasing the exciton bandwidth increases the intensity of the 0-0 transition and decreases that of the 0-1 transition, i.e., the intensity ratio increases with decreasing $W$. The strength of interchain interactions and the exciton bandwidth are closely related to the conjugation length of the polymer. When the conjugation length is longer than the intermolecular separation, as is generally the case in polymers, quantumchemical calculations have shown that the exciton bandwidth decreases as the conjugation length increases. ${ }^{1,46,47}$ This is because for long conjugation lengths the point dipole approximation breaks down and there is a tendency for positive and negative contributions to the atomic transition densities to cancel each other, leading to a reduction of the interaction energy/exciton bandwidth. 
Within this model we can now interpret the subtle variations in the absorption spectra as a function of MW in terms of variations of the intrachain conjugation length. The results of chloroform spun films [Fig. 7(a)] reveal that exciton bandwidth increases and conjugation length decreases with MW as a result of disorder frozen during rapid film deposition [see Fig. 4(a)]. The MW independence of the mobility might reflect the interplay between the beneficial effect of increased chain lengths and larger number of tie molecules at higher MW (Ref. 13) and the adverse effect of reduced conjugation length. For the TCB spin-cast films the low intensity of the 0-0 transition with respect to the $0-1$ transition in low MW samples suggests a short conjugation length, and a comparatively large $W$. This is consistent with the presence of chain ends, chemical defects and impurities within the nanoribbon lamellae as postulated above. In the intermediate MW range the intensity of the $0-0$ transition is highest, suggesting that these samples have the weakest exciton coupling, the longest conjugation length, and the highest crystal quality. For even higher MW the intensity of the 0-0 transition decreases again indicating a shortening of conjugation length, higher conformational disorder and reduced crystallinity. This interpretation of the optical absorption spectra is fully consistent with the microstructural model postulated in Fig. 6.

The emission spectra of TCB films at low temperature $(10 \mathrm{~K})$ exhibit a similar systematic dependence on MW (Fig. $8)$. The intensity ratio of $0-0$ emission $(\sim 1.86 \mathrm{eV})$ to $0-1$ emission $(\sim 1.68 \mathrm{eV})$ is minimum in low $\mathrm{MW}$ polymers $(20 \mathrm{kD})$ and reaches a maximum in intermediate MW polymers (42 kD), and then reduces again in high MW polymer $(76 \mathrm{kD})$. This trend is analogous to that observed in the absorption spectra. We emphasize that although the spectral differences in the absorption and emission spectra may appear small the spectral evolution as a function of molecular weight was very systematic and consistent among all the MW samples investigated. It was observed reproducibly in different experimental runs in both spin cast as well as dropcast films from TCB solution, and was independent of the film thickness within a range of $20-50 \mathrm{~nm}$. In order not to compromise the clarity of the figure we only show representative spectra in Figs. 7 and 8. Based on Spano's model this behavior can be interpreted as a combined effect of a variation of exciton coupling/conjugation length and degree of disorder as a function of MW. From the model in Ref. 43 we can derive a simple expression for the ratio of the $0-0$ to $0-1$ emission peaks, which is expected to be valid in the limit of spatially uncorrelated disorder [ $(\beta=0)$, as defined in Ref. 43]

$$
\frac{I_{e}^{0-0}}{I_{e}^{0-1}} \approx \frac{\left(1-0.24 W / \omega_{0}\right)^{2}}{2 e^{-2}\left(1-0.39 W / \omega_{0}\right)^{2}} \frac{\sigma^{2}}{W^{2}} .
$$

Here $\sigma$ is the half width at $1 / e$ of the Gaussian distribution of site-energy disorder, and we have also assumed that the Huang-Rhys (HR) factor $\lambda^{2}=1 .{ }^{48,49}$ It is clear from Eq. (2) that smaller exciton bandwidth and/or increased disorder yields more intense $0-0$ emission relative to $0-1$ emission. To estimate $W$ and $\sigma$ as a function of MW we use the experimental absorption spectra to obtain a value of $W / \omega_{0}$ for different MW polymers from Eq. (1). We then substitute the

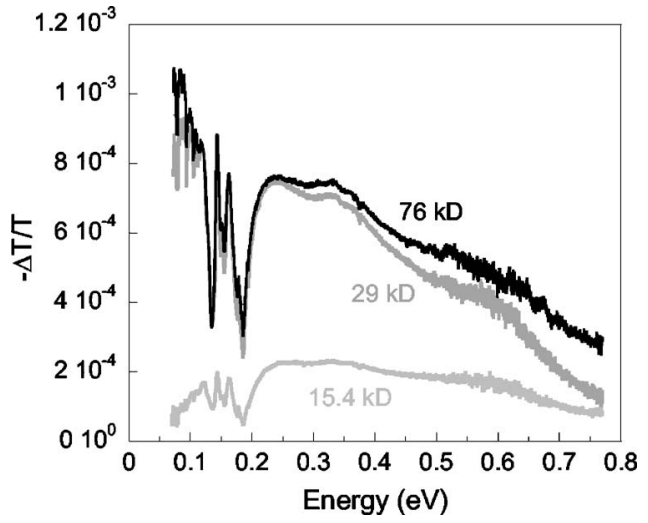

FIG. 9. Charge modulation spectra in the mid-infrared spectral range of TCB spin-cast films for different MW. The spectra were obtained by subtracting the infrared transmission spectra of the device structure taken at 10 and $-30 \mathrm{~V}$.

experimental 0-0 to 0-1 emission peak ratio and the value of $W / \omega_{0}$ into Eq. (2) to obtain a value of $\sigma / \omega_{0}$. This analysis (see the inset of Fig. 8) shows that the exciton coupling $W$ varies strongly with MW exhibiting a minimum value for intermediate molecular weights. The disorder parameter $\sigma$ exhibits only a comparatively weak variation, although it also appears to be minimum for intermediate molecular weights. This variation of $\sigma$ with MW is consistent with the one expected from a comparison of the vibronic linewidth of the 0-0 transition as a function of MW. We note that the values of $\sigma$ extracted based on this simplified analysis are somewhat smaller by a factor of 1-2 than those obtained by fitting the line shape of vibrational transitions with a Gaussian. Within the model this disagreement is expected to be diminished as a more realistic assumption of spatially correlated disorder is considered $(\beta>0)$, since larger values of $\sigma$ are required to compensate the stronger suppression of $0-0$ to $0-1$ emission peak ratio due to the increased $\beta$.

The observed dependence of the exciton bandwidth, and disorder parameter on MW is fully consistent with the structural model proposed above. Polymers of intermediate MW have the highest crystalline quality and longest conjugation length leading to the smallest exciton bandwidth and disorder parameter. In the highest MW polymers the conjugation length is slightly reduced, and exciton bandwidth increased as a result of kinetically induced disorder. Crystallites in lower MW samples contain a higher density of structural defects, such as those associated with chain ends, leading to much shorter conjugation length, larger exciton bandwidth and disorder parameter.

Finally, we have also investigated the MW dependence of the spectroscopic properties of charged polarons using charge modulation spectroscopy [(CMS), Fig. 9]..$^{50}$ The CMS technique measures changes in the optical transmission of an operational field-effect transistor (FET) upon gate-voltage induced modulation of the charge concentration in the accumulation layer of the FET as a function of wavelength. In this way it yields information about the characteristic chargeinduced absorption spectrum of the polaronic charge carriers that carry the current in the FET. Previous CMS experiments on regioregular $\mathrm{P} 3 \mathrm{HT}$ have revealed a pronounced low- 
energy charge transfer (CT) transition in the midinfrared spectral region. 6,50 This together with the appearance of an additional charge-induced absorption in the visible spectral range which is symmetry forbidden in isolated chains was interpreted as evidence for delocalization of polarons over a number of neighboring chains as a result of interchain interactions. $^{6,50}$ This interpretation of the CMS spectra was based on the theoretical work by Blackman and Sabra who predicted that for interchain coupling strength on the order of $0.15 \mathrm{eV}$ the $1 \mathrm{D}$ polaron abruptly delocalizes, ${ }^{51}$ and was confirmed by quantum chemical calculations of the chargeinduced absorption spectra of molecular aggregates. ${ }^{52}$ Similar results were obtained by Österbacka et al. based on photo-induced absorption (PIA) measurements on $\mathrm{P} 3 \mathrm{HT},{ }^{53-56}$ who showed that increased interchain coupling leads to a splitting of the single chain polaron energy levels by $2 \Delta$ and a red shift of the lower energy polaron band (called $\mathrm{DP}_{1}$ in Ref. 53). The $\mathrm{DP}_{1}$ feature observed in the PIA spectra of Österbacka is of the same origin as the CT transition observed in the CMS spectra reported here.

The delocalization of polarons as a result of interchain interactions and the associated appearance of the CT transition can also be understood in the equivalent framework of Marcus-Hush electron transfer theory ${ }^{57}$ describing the transfer of electrons between neighboring molecules in the presence of strong electron-lattice interactions. The electron transfer process is governed by two main parameters: The relaxation energy $\lambda$, which is twice the polaron binding energy, measures the energy lowering which charged molecules can achieve by adopting a relaxed conformation as a result of the electron-lattice coupling. The transfer integral $t$ is a measure of the strength of the interchain coupling of the electronic wave functions on neighboring molecules. In the weak coupling case $(\lambda>2 t)$ the lower adiabatic potential surface has a number of minima, and the charge is localized on an individual molecule. Under such conditions a charge transfer optical transition is observed centered at an energy $\hbar \omega_{\mathrm{CT}}$ $=\lambda$. The regime of delocalized polarons corresponds to the strong coupling case $(\lambda<2 t)$ in which the lower adiabatic potential surface has only one minimum and the charge is delocalized over a certain number of neighboring molecules. In this case an optical charge transfer transition can also be observed, but it is centered not around $\lambda$ but around $\hbar \omega_{\mathrm{CT}}$ $=2 t$, i.e., it corresponds to the splitting of the single-chain polaron levels by the interchain interaction.

In intermediate MW samples with mobilities $>0.05 \mathrm{~cm}^{2} / \mathrm{V} \mathrm{s}$ we observe an intense CT transition centered around $0.1 \mathrm{eV}$ (Fig. 9). In the highest mobility, highest MW samples the transition is similarly intense and appears to peak at slightly lower energies below $0.08 \mathrm{eV}$, which is the low energy cutoff of our experimental setup. In contrast, in the low MW samples with mobilities less than $10^{-2} \mathrm{~cm}^{2} / \mathrm{V} \mathrm{s}$ a much less intense CT transition is observed, and the transition peaks at significantly higher energies on the order of $0.3 \mathrm{eV}$. The position and intensity of the CT transition appears to be very directly correlated with the field-effect mobility. We have shown that in high mobility P3HT the strong coupling situation applies. ${ }^{52}$ This allows us to estimate the transfer integral for interchain electron transfer to be on the order of $t=0.05-0.08 \mathrm{eV}$ for intermediate MW. It is more difficult to obtain information about $\lambda$ in this regime, ${ }^{58}$ but we can give an upper limit estimate for $\lambda<2 t$. The lower intensity of the CT transition indicates a lower degree of interchain polaron delocalization in the low MW samples. A plausible, but somewhat speculative explanation for the reduced intensity and higher energy of the CT transition in the low MW samples is that due to the enhanced disorder and shorter conjugation length in these samples the weak coupling regime might apply. Such a crossover behavior between localized and delocalized polarons as a function of MW would provide an intriguing microscopic explanation for the observed rapid increase of mobility with MW below $15-20 \mathrm{kD}$ (see Fig. 1). Based on this assumption one could estimate the relaxation energy in low MW samples to be on the order of $0.2-0.3 \mathrm{eV}$. A more detailed quantitative analysis of the CMS spectra and the correlation between relaxation energy and temperature dependent transport data will be published elsewhere. ${ }^{59}$

\section{DISCUSSION AND CONCLUSIONS}

We have presented for the first time a systematic investigation of interchain interaction effects on the properties of neutral excitons and charged polarons in conjugated polymers as a function of MW. In order to interpret the spectroscopic data correctly it is necessary to unravel the complex polymer microstructure and have a clear understanding of the variations in chain folding that occur when varying the MW. Based on XRD, AFM and electron microscopy we have proposed a structural model for highly crystalline P3HT as a function of MW. Only for the very lowest MW ( $3 \mathrm{kD})$ extended-chain nanorod crystals are formed. As MW exceeds above $15 \mathrm{kD}$, morphology and mobility are shown to be very sensitive to the processing conditions. Under rapid deposition conditions the formation of well-defined nanorods is not observed for any of the MWs, but only comparatively amorphous microstructures are formed in which a bimodal orientation of the conjugated lamella with respect to the substrate is present. This limits the mobility to relatively low values around $10^{-2} \mathrm{~cm}^{2} / \mathrm{V} \mathrm{s}$ independent of MW because it requires charges to hop across the insulating side chains. In contrast, slow deposition by spin-casting from high boiling-point solvents allows the polymer chains to self-organize into welldefined, and highly crystalline nanoribbons with unimodal in-plane orientation of the direction of $\pi$ - $\pi$ stacking along the long axis of the nanoribbons. Unlike the MWindependent mobility in rapidly deposited films, mobility increases sharply from low MW (15-20 kD) to intermediate MW (29-52 kD), and becomes saturated slightly above $0.1 \mathrm{~cm}^{2} / \mathrm{V} \mathrm{s}$ for sufficiently high MW (>52 kD).

The crystalline quality is lowest in the low MW regime, which is attributed at least partly to the higher population of chain ends and associated defects inside the nanoribbons. In an intermediate MW range (29-52 kD) the crystalline quality is highest as longer chains can fold into the nanoribbons without producing chain end defects inside the nanoribbons. We believe that this improvement in crystalline quality inside the nanoribbons is primarily responsible for the rapid increase of mobility from low to intermediate MW. In the high 
MW regime $(>52 \mathrm{kD})$ one might expect the mobility to increase further due to the increased density of tie molecules between nanoribbons, as well as their preferred parallel arrangement with respect to each other. However, this expected benefit is partly offset by an increased degree of disorder, which may be either an intrinsic property of long chains (for example due to branching) or arising from slower crystallization kinetics. The combination of these two effects limits the mobility at values of $0.1 \mathrm{~cm}^{2} / \mathrm{V} \mathrm{s}$ in the high $\mathrm{MW}$ samples.

Based on this microstructural model of chain folding as a function of MW we can explain the evolution of the optical absorption and emission spectra of P3HT with MW. In the intermediate MW range where the conjugation length is highest we observe the smallest exciton bandwidth $W$ and smallest disorder parameter $\sigma$. The high MW samples have slightly increased exciton bandwidth and disorder presumably due to a slowing of the timescale required for crystallization. In contrast, the low MW samples with shorter conjugation length have much larger exciton bandwidth and disorder parameter. To the best of our knowledge this constitutes the first experimental observation in a conjugated polymer of the lowering of the exciton bandwidth with increasing conjugation length. This was predicted theoretically for conjugated oligomer model systems. ${ }^{1}$

We have also obtained experimental evidence that charged polarons are delocalized over several chains for intermediate and high MW, while for low MWs the degree of polaron delocalization is significantly lower as evidenced by a much reduced intensity of the charge transfer chargeinduced transitions in the mid infrared. This provides a mi- croscopic explanation for the observed rapid increase of mobility from low to intermediate MWs. The clear correlation between the degree of polaron delocalization in the polymer microcrystals detected spectroscopically on a molecular scale and the field-effect mobility measured on a mesoscopic scale suggests that in the high mobility transport regime ( $\mu$ $=0.1 \mathrm{~cm}^{2} / \mathrm{V} \mathrm{s}$ ) it is primarily microscopic factors such as the crystal quality within the polymer microcrystals that limit the transport, and not grain boundaries between individual nanofibers. This is consistent with our observation of only a weak channel length dependence of the mobility (Fig. 2), the absence of well-defined potential barriers associated with grain boundaries in scanning kelvin probe potential imaging $^{60}$ and with previous measurements on individual P3HT nanofibers, which showed no evidence of an enhanced field-effect mobility in spite of grain boundary effects being eliminated in such experiments. ${ }^{24,35}$ Further breakthroughs to achieve even higher mobilities in crystalline polymers such as P3HT require unique processes to better control the various factors that determine the crystal growth and the chain conformation, and to improve the crystalline quality and eliminate structural and chemical defects in high MW P3HT nanoribbon crystals.

\section{ACKNOWLEDGMENTS}

It is a pleasure to acknowledge stimulating discussions with Jerome Cornil and David Beljonne, and funding from the Engineering and Physical Sciences Research Council (EPSRC) under the "Carbon-based Electronics-A national consortium" program.
*Corresponding author. Email address: hs220@phy.cam.ac.uk

${ }^{1}$ D. Beljonne, J. Cornil, R. Silbey, P. Millié, and J. L. Brédas, J. Chem. Phys. 112, 4749 (2000).

${ }^{2}$ G. H. Gelinck, H. E. A. Huitema, E. V. Veenendaal, E. Cantatore, L. Schrijnemakers, J. B. P. H. Van der Putten, T. C. T. Geuns, M. Beenhakkers, J. B. Giesbers, B.-H. Huisman, E. J. Meijer, E. M. Benito, F. J. Touwslager, A. W. Marsman, B. J. E. Van Rens, and D. M. de Leeuw, Nat. Mater. 3, 106 (2004).

${ }^{3}$ C. D. Dimitrakopoulos and D. J. Mascaro, IBM J. Res. Dev. 45, 11 (2001).

${ }^{4}$ W. Clemens, W. Fix, J. Ficker, A. Knobloch, and A. Ullmann, J. Mater. Res. 19, 1963 (2004).

${ }^{5}$ H. Sirringhaus, Adv. Mater. (Weinheim, Ger.) 17, 2411 (2005).

${ }^{6}$ H. Sirringhaus, P. J. Brown, R. H. Friend, M. M. Nielsen, K. Bechgaard, B. M. W. Langeveld-Voss, A. J. H. Spiering, R. A. J. Janssen, E. W. Meijer, P. Herwig, and D. M. de Leeuw, Nature (London) 401, 685 (1999).

${ }^{7}$ H. Sirringhaus, N. Tessler, and R. H. Friend, Science 280, 1741 (1998).

${ }^{8}$ D. H. Kim, Y. D. Park, Y. Jang, H. Yang, Y. H. Kim, J. I. Han, D. G. Moon, S. Park, T. Chang, C. Chang, M. Joo, C. Y. Ryu, and K. Cho, Adv. Funct. Mater. 15, 77 (2005).

${ }^{9}$ H. Yang, T. J. Shin, L. Yang, K. Cho, C. Y. Ryu, and Z. Bao, Adv. Funct. Mater. 15, 671 (2005).
${ }^{10}$ D. W. Kim, Y. D. Park, Y. Jang, S. Kim, and K. Cho, Macromol. Rapid Commun. 26, 834 (2005).

${ }^{11}$ J.-F. Chang, B. Sun, D. W. Breiby, M. M. Nielsen, T. I. Sölling, M. Giles, I. McCulloch, and H. Sirringhaus, Chem. Mater. 16, 4772 (2004).

${ }^{12}$ G. Wang, J. Swensen, D. Moses, and A. J. Heeger, J. Appl. Phys. 93, 6137 (2003).

${ }^{13}$ R. J. Kline, M. D. McGehee, E. N. Kadnikova, J. Liu, and J. M. J. Fréchet, Adv. Mater. (Weinheim, Ger.) 15, 1519 (2003).

${ }^{14}$ R. J. Kline, M. D. McGehee, E. N. Kadnikova, J. Liu, J. M. J. Fréchet, and M. F. Toney, Macromolecules 38, 3312 (2005).

${ }^{15}$ A. Zen, J. Pflaum, S. Hirschmann, W. Zhuang, F. Jaiser, U. Asawapirom, J. P. Rabe, U. Scherf, and D. Neher, Adv. Funct. Mater. 14, 757 (2004).

${ }^{16}$ G. Koller, B. Falk, C. Weller, M. Giles, and I. McCulloch, Process of preparing regioregular poly- (3-substituted) thiophenes., PCT Int. Appl., WO2005014691 (2005).

${ }^{17}$ L. Bürgi, T. J. Richards, R. H. Friend, and H. Sirringhaus, J. Appl. Phys. 94, 6129 (2003).

${ }^{18}$ B. W. Warren, X-ray Diffraction (Dover, New York, 1969).

${ }^{19}$ E. J. Samuelsen and J. Mårdalen, in Handbook of Organic Conductive Molecules and Polymers; Vol. 3, Conductive Polymers: Spectroscopy and Physical Properties, 1st ed., edited by H. S. Nalwa (Wiley, Weinheim, 1997). 
${ }^{20}$ T. J. Prosa, M. J. Winokur, J. Moulton, P. Smith, and A. J. Heeger, Macromolecules 25, 4364 (1992).

${ }^{21}$ L. Bürgi, T. Richards, M. Chiesa, R. H. Friend, and H. Sirringhaus, Synth. Met. 146, 297 (2004).

${ }^{22}$ L. Bürgi, H. Sirringhaus, and R. H. Friend, Appl. Phys. Lett. 80, 2913 (2002).

${ }^{23}$ E. J. Meijer, G. H. Gelinck, E. van Veenendaal, B.-H. Huisman, D. M. de Leeuw, and T. M. Klapwijk, Appl. Phys. Lett. 82, 4576 (2003).

${ }^{24}$ M. Mas-Torrent, D. den Boer, M. Durkut, P. Hadley, and A. P. H. J. Schenning, Nanotechnology 15, S265 (2004).

${ }^{25}$ M. L. Chabinyc, J.-P. Lu, R. A. Street, Y. Wu, P. Liu, and B. S. Ong, J. Appl. Phys. 96, 2063 (2004).

${ }^{26}$ M. Van der Auweraer, F. C. De Schryver, P. M. Borsenberger, and H. Bässler, Adv. Mater. (Weinheim, Ger.) 6, 199 (1994).

${ }^{27}$ P. W. M. Blom, M. J. M. de Jong, and M. G. van Munster, Phys. Rev. B 55, R656 (1997).

${ }^{28}$ K. Waragai, H. Akimichi, S. Hotta, H. Kano, and H. Sakaki, Phys. Rev. B 52, 1786 (1995).

${ }^{29}$ C. Goh, R. J. Kline, M. D. McGehee, E. N. Kadnikova, and J. M. J. Fréchet, Appl. Phys. Lett. 86, 122110 (2005).

${ }^{30}$ H. J. Fell, E. J. Samuelsen, J. Als-Nielsen, G. Grübel, and J. Mårdalen, Solid State Commun. 94, 843 (1995).

${ }^{31}$ K. E. Aasmundtveit, E. J. Samuelsen, M. Guldstein, C. Steinsland, O. Flornes, C. Fagermo, T. M. Seeberg, L. A. A. Pettersson, O. Inganäs, R. Feidenhans'1, and S. Ferrer, Macromolecules 33, 3120 (2000).

${ }^{32}$ D. W. Breiby and E. J. Samuelsen, J. Polym. Sci., Part B: Polym. Phys. 41, 2375 (2003).

${ }^{33}$ T. Bjørnholm, T. Hassenkam, D. R. Greve, R. D. McCullough, M. Jayaraman, S. M. Savoy, C. E. Jones, and J. T. McDevitt, Adv. Mater. (Weinheim, Ger.) 11, 1218 (1999).

${ }^{34}$ K. J. Ihn, J. Moulton, and P. Smith, J. Polym. Sci., Part B: Polym. Phys. 31, 735 (1993).

${ }^{35}$ J. A. Merlo and C. D. Frisbie, J. Polym. Sci., Part B: Polym. Phys. 41, 2674 (2003)

${ }^{36}$ X. Yang, J. Loos, S. C. Veenstra, W. J. H. Verhees, M. M. Wienk, J. M. Kroon, M. A. J. Michels, and R. A. J. Janssen, Nano Lett. 5, 579 (2005).

${ }^{37}$ N. Kiriy, E. Jähne, H.-J. Adler, M. Schneider, A. Kiriy, G. Gorodyska, S. Minko, D. Jehnichen, P. Simon, A. A. Fokin, and M. Stamm, Nano Lett. 3, 707 (2003).
${ }^{38}$ S. Malik and A. K. Nandi, J. Polym. Sci., Part B: Polym. Phys. 40, 2073 (2002).

${ }^{39}$ B. Wunderlich, Macromolecular Physics; Vol. 1, Crystal Structure, Morphology, Defects (Academic Press, New York, 1973).

${ }^{40}$ L. Mandelkern, J. M. Price, M. Gopalan, and J. G. Fatou, J. Polym. Sci., Part A-2 4, 385 (1966).

${ }^{41}$ B. Wunderlich and T. Davidson, J. Polym. Sci., Part A-2 7, 2043 (1969).

${ }^{42}$ P. J. Brown, D. S. Thomas, A. Köhler, J. S. Wilson, J.-S. Kim, C. M. Ramsdale, H. Sirringhaus, and R. H. Friend, Phys. Rev. B 67, 064203 (2003).

${ }^{43}$ F. C. Spano, J. Chem. Phys. 122, 234701 (2005).

${ }^{44}$ O. J. Korovyanko, R. Österbacka, X. M. Jiang, Z. V. Vardeny, and R. A. J. Janssen, Phys. Rev. B 64, 235122 (2001).

${ }^{45}$ G. Louarn, J.-Y. Mevellec, J. P. Buisson, and S. Lefrant, Synth. Met. 55-57, 587 (1993).

${ }^{46}$ J. Cornil, D. A. Dos Santos, X. Crispin, R. Silbey, and J. L. Brédas, J. Am. Chem. Soc. 120, 1289 (1998).

${ }^{47}$ E. S. Manas and F. C. Spano, J. Chem. Phys. 109, 8087 (1998).

${ }^{48}$ D. Oelkrug, H.-J. Egelhaaf, J. Gierschner, and A. Tompert, Synth. Met. 76, 249 (1996).

${ }^{49}$ J. Gierschner, H.-J. Egelhaaf, and D. Oelkrug, Synth. Met. 84, 529 (1997)

${ }^{50}$ P. J. Brown, H. Sirringhaus, M. Harrison, M. Shkunov, and R. H. Friend, Phys. Rev. B 63, 125204 (2001).

${ }^{51}$ J. A. Blackman and M. K. Sabra, Phys. Rev. B 47, 15437 (1993).

${ }^{52}$ D. Beljonne, J. Cornil, H. Sirringhaus, P. J. Brown, R. H. Friend, and J. L. Brédas, Adv. Funct. Mater. 11, 229 (2001).

${ }^{53}$ R. Österbacka, C. P. An, X. M. Jiang, and Z. V. Vardeny, Science 287, 839 (2000).

${ }^{54}$ R. Österbacka, X. M. Jiang, C. P. An, B. Horovitz, and Z. V. Vardeny, Phys. Rev. Lett. 88, 226401 (2002).

${ }^{55}$ R. Österbacka, C. P. An, X. M. Jiang, and Z. V. Vardeny, Synth. Met. 116, 317-320 (2001).

${ }^{56}$ X. M. Jiang, C. P. An, R. Österbacka, and Z. V. Vardeny, Synth. Met. 116, 203 (2001).

${ }^{57}$ R. A. Marcus, J. Chem. Phys. 24, 966 (1956).

${ }^{58}$ V. Coropceanu, M. Malagoli, J. M. Andre, and J. L. Brédas, J. Am. Chem. Soc. 124, 10519 (2002).

${ }^{59}$ J.-F. Chang and H. Sirringhaus (unpublished).

${ }^{60} \mathrm{M}$. Chiesa and H. Sirringhaus (unpublished). 